\title{
A666-conjugated nanoparticles target prestin of outer hair cells preventing cisplatin-induced hearing loss
}

This article was published in the following Dove Press journal: International Journal of Nanomedicine

\author{
Xueling Wang ${ }^{1-3, *}$ \\ Yuming Chen ${ }^{1-3, *}$ \\ Yong $\mathrm{TaO}^{1-3}$ \\ Yunge $\mathrm{Gao}^{1-3}$ \\ Dehong $\mathrm{Yu}^{1-3}$ \\ $\mathrm{Hao} \mathrm{Wu}^{1-3}$
}

'Department of OtolaryngologyHead and Neck Surgery, Shanghai Ninth People's Hospital, Shanghai Jiao Tong University School of Medicine, Shanghai, People's Republic of China; ${ }^{2}$ Ear Institute, Shanghai Jiao Tong University School of Medicine, Shanghai, People's Republic of China; ${ }^{3}$ Shanghai Key Laboratory of Translational Medicine on Ear and Nose Diseases, Shanghai, People's Republic of China

*These authors contributed equally to this work
Correspondence: Dehong Yu; Hao Wu Department of Otolaryngology-Head and Neck Surgery, Shanghai Ninth People's Hospital, Shanghai Jiao Tong University School of Medicine, Shanghai, 2000I I, People's Republic of China Tel +862163288830 Email dehongyu@I26.com; wuhao@ sh9hospital.org
Background: The delivery of treatment agents to inner ear with drug delivery system (DDS) has been under investigation to overcome the limitations of the conventional therapeutic agents in curing or alleviating the cisplatin ototoxicity.

Methods: In the present study, a novel targeted dexamethasone(DEX)-loaded DDS, A666-DEX-NP, was constructed for prevention from cisplatin-induced hearing loss. A666-(CLEPRWGFGWWLH) peptides specifically bind to prestin, which is limited to the outer hair cells (OHCs). HEI-OC1 and cisplatin-treated guinea pigs ( $12 \mathrm{mg} / \mathrm{kg}$, intraperitoneal) were used as in vitro and in vivo models for investigating the targeting and protective efficiency against cisplatin.

Results: As expected, compared to A666-unconjugated nanoparticles (NP), A666-conjugated coumarin 6-labeled NP showed active targeting to OHCs. Furthermore, A666-coumarin 6-labeled NP could be significantly internalized by HEI-OC1 cells via the A666-prestin interaction. This facilitated the uptake of cells pretreated with A666-DEX-NP, followed by the cisplatin-treated group, which led to enhanced cell viability, reduced apoptotic properties, and decreased reactive oxygen species levels as compared to cells pretreated with DEX or DEX-NP, 4 hours in advance of cisplatin treatment. In cisplatin-treated guinea pigs, pretreatment with A666-DEX-NP effectively preserved $\mathrm{OHCs}$ and showed significant hearing protection at 4,8 , and $16 \mathrm{kHz}$ as compared to pretreatment with saline, DEX, or DEX-NP formulation.

Conclusion: This OHC-targeting DDS provides a novel strategy for DEX application that can be potentially used to combat cisplatin ototoxicity.

Keywords: A666 peptide, dexamethasone, prestin, cisplatin ototoxicity

\section{Introduction}

Cisplatin (CDDP) is extensively used as an antineoplastic regimen for the treatment of cancers ranging from the ovarian, non-small-cell lung, and head and neck to testicular. However, its therapeutic outcomes are associated with ototoxicity, ${ }^{1}$ neurotoxicity, ${ }^{2}$ and nephrotoxicity. ${ }^{3}$ Hydration increases diuresis, and in turn, can reduce the nephrotoxicity. However, ototoxicity is bilateral and permanent, and can severely impact the quality of life of the cancer patients. Approximately, $40 \%-80 \%$ adult cancer patients and $90 \%$ pediatric patients ${ }^{4,5}$ who underwent CDDP treatment have suffered from severe hearing loss. Since the damaged mechanoreceptor hair cells cannot regenerate in humans and other mammals, ${ }^{6,7}$ exploring new agents or strategies for preventing the damage to hair cells from CDDP is essential.

The cellular and molecular mechanism underlying CDDP-induced cytotoxicity is still controversial; however, it includes damage of DNA, generation of reactive oxygen species (ROS), and the accumulated release of proinflammatory cytokines, 
including TNF- $\alpha$, IL- $1 \beta$, and NF- $\kappa$ B into the cytoplasm. ${ }^{8}$ Aiming at different mechanistic pathways, otoprotective clinical candidates are designed to combat this ototoxicity, including dexamethasone (DEX), sodium thiosulfate (STS), acetylcysteine, D-methionine, and amifostine. ${ }^{8}$ Nevertheless, neither of these drugs have yet been approved by the US Food and Drug Administration to prevent CDDP-induced hearing loss. Instead of seeking a new otoprotective drug, developing an active targeting drug delivery system (DDS), which could serve as a specific carrier of the drugs to the particular cell, has gained increasing attention.

Outer hair cells (OHCs) are the sensory receptors which are responsible for amplifying sound signals. Thus, the damage or loss of OHCs is the common cause of CDDP ototoxicity. ${ }^{9,10}$ Targeting otoprotective agents to OHCs in the organ of Corti by nanomedicine strategies is crucial for curing ototoxicity relative to the pathology of OHCs. Prestin is one of the vital molecular motors and is closely involved in electromechanical transduction. ${ }^{11-13}$ It is specifically expressed in the lateral membrane of OHCs of the cochlea. Recently, a peptide designated, A666 (CLEPRWGFGWWLH), isolated from 3 rounds of sequential phage display was proved to bind the extracellular loops of prestin with enhanced affinity and specificity. ${ }^{14}$ The selective binding properties of A666 to prestin were demonstrated by flow cytometry in Chinese hamster ovary cells. Moreover, PEG6K-b-PCL19K polymersomes labeled with this peptide actively accumulated on the $\mathrm{OHCs}$ in a rat cochlear explant model. ${ }^{14}$ Thus, we proposed that A666 peptide has the potential to be chosen as an efficient ligand for nano-based DDS, which can actively target the OHCs through the binding of A666 peptide to prestin.

DEX exerts hearing protection against CDDP ototoxicity through various mechanisms, including enhancing antioxidant enzymes, inhibiting apoptosis, and reducing proinflammatory cytokines. ${ }^{15,16}$ However, systemic administration of DEX led to antitumor interference with CDDP due to the downregulation of apoptosis gene. ${ }^{17}$ Moreover, intratympanic administration of DEX has yielded conflicting results, owing to the elimination of the drug through the eustachian tube, rapid clearance of drugs, and nonspecific distribution in the inner ear. Nanotechnologies have advantages such as sustained release and specific targeting, which have attracted great attention in the past decades for delivering agents to the inner ear. ${ }^{18-20}$ Unlike medical devices or hydrogels, the nanoparticulate system offers the possibility of performing surface functionalization, which would not be possible on therapeutic molecules alone without risking a loss of molecular function.
In this study, we constructed a novel OHC-targeting nanotechnology DDS consisting of A666 peptide-conjugated nanoparticles (NPs) loaded with DEX (designated A666DEX-NP), and aimed to investigate the possibility of A666-DEX-NP restoring CDDP-induced hearing loss. The maleimide-thiol coupling technique, coupling the SHterminal amine of the peptide to the maleimide groups of poly(ethylene glycol) (PEG) protruding on the surface of the NPs, was used to decorate the NPs with the A666 peptide. The active OHC targeting ability of A666-DEX-NP was achieved by a unique OHCs homing peptide (A666) that is conjugated to their surface. In order to achieve clinical translation, a US Food and Drug Administration-approved biomaterial, poly(lactide) (PLA)-based formulation was chosen in the present study owing to its high biocompatibility and biodegradable characteristic. The current results indicated that A666-DEX-NP accumulated on OHCs and were significantly internalized by HEI-OC1 cells via the A666-prestin interaction. This facilitated the uptake of cells pretreated with A666-DEX-NP that led to enhanced cell viability, reduced apoptotic properties, and decreased ROS levels as compared to cells pretreated with DEX or DEX-NP in CDDP-treated HEI-OC1 cell model. Furthermore, A666DEX-NP significantly improved the protective efficacy of DEX against CDDP-induced ototoxicity at 4, 8 , and $16 \mathrm{kHz}$ frequencies in CDDP-treated guinea pig model by round window membrane (RWM) administration.

\section{Materials and methods Materials, cell culture, and animals}

Maleimide PEG-PLA (Mal-PEG-PLA, molecular weight [MW] $50 \mathrm{kDa}$ ) and methoxy (polyethylene glycol)poly(lactide) (mPEG-PLA, MW $50 \mathrm{kDa}$ ) were purchased from Daigang Biomaterial Co., Ltd. (Ji'nan, People's Republic of China). A666 peptide (CLEPRWGFGWWLH) was synthesized by GL Biochem Ltd. (Shanghai, People's Republic of China), coumarin 6 was purchased from Aldrich (St Louis, MO, USA), and DEX from Bristol-Myers Squibb (New York, NY, USA). DAPI was purchased from Invitrogen (Carlsbad, CA, USA). CDDP was purchased from Aladdin (Shanghai, People's Republic of China). Cell Counting Assay Kit-8 (CCK-8) was procured from Dojindo Molecular Technologies (Kumamoto, Japan).

HEI-OC1 (generated at House Ear Institute, Los Angeles, CA, USA), an inner ear cell line derived from the auditory organ of transgenic mouse Immortomouse ${ }^{\mathrm{TM}}$, is a potential tool to study the ototoxic mechanisms of ototoxic drugs in vitro. ${ }^{21} \mathrm{HEI}-\mathrm{OC} 1$ was maintained in high-glucose Dulbecco's Modified Eagle's Medium containing 10\% fetal 
bovine serum (FBS; Gibco, Waltham, MA, USA) without antibiotics at $33^{\circ} \mathrm{C}$ and $10 \% \mathrm{CO}_{2}$, as described previously. ${ }^{21}$

Male guinea pigs (4-6-week-old, weighing about $250 \mathrm{~g}$ ) were purchased from the Shanghai Laboratory Animal Center (Shanghai, People's Republic of China). All animals were allowed free access to standard rat chow and water. The animal experiments were approved by the Ethics Committee of Shanghai Jiao Tong University, School of Medicine. Animal experiments complied with the 3R principles (Reduction, Replacement, and Refinement).

\section{Preparation of A666-DEX-NP}

Emulsion and solvent extraction/evaporation method and the classic preparation technology of NP were used to prepare DEX-NP. A666-DEX-NP was fabricated using a follow-up peptide surface conjugation (Figure 1). Briefly, $5 \mathrm{mg}$ DEX was solubilized in a mixture of Mal-PEG-PLA and mPEG-PLA $(1 / 9, \mathrm{w} / \mathrm{w})$ in $1 \mathrm{~mL} \mathrm{CH}_{2} \mathrm{Cl}_{2}$ solution. The obtained oil phase was added into $3 \mathrm{~mL}$ of sodium cholate solution $\left(\mathrm{C}_{24} \mathrm{H}_{39} \mathrm{NaO}_{5}, 1 \%\right.$, w/v), followed by sonication for 30 seconds at $220 \mathrm{~W}$ (Scientz Biotechnology Co. Ltd., Ningbo, People's Republic of China). The emulsion was mixed with $36 \mathrm{~mL}$ of $0.5 \% \mathrm{C}_{24} \mathrm{H}_{39} \mathrm{NaO}_{5}$ solution, followed by rotary evaporation under reduced pressure to remove the organic solvent. The DEX-NPs were collected by centrifugation at $11,000 \times g$ for 30 minutes.

A666-decorated NP were prepared as following: DEX-NP were incubated with A666 peptide (Mal-PEG-PLA/ A666, 1:3, mol/mol). About $30 \mathrm{mg}$ DEX-NP was dispersed in $10 \mathrm{~mL}$ of $0.05 \mathrm{M} \times$ HEPES (pH 7.4) and placed at room temperature for 6 hours. The A666-DEX-NP was harvested by centrifugation at $11,000 \times g$ for 30 minutes and lyophilized for further use. The coumarin 6-labeled NP preparation was similar to that as A666-DEX-NP, except that $0.05 \%$ (w/v) coumarin 6 was used instead of DEX. To verify the successful decoration of A666 peptides, the unconjugated concentration of A666 peptide in the supernatant after the reaction was quantified by CBQCA Protein Quantitation Kit (Thermo Fisher Scientific, Waltham, MA, USA).

A666 surface density $(S)$, the number of A666 peptides conjugated per NP, was obtained by dividing the number of A666 peptides by the calculated average number $(\mathrm{N})$ of nanoparticles using the method described by Olivier et al..$^{22}$ The average distance $(D)$ between 2 neighboring PEG chains linked to A666

A

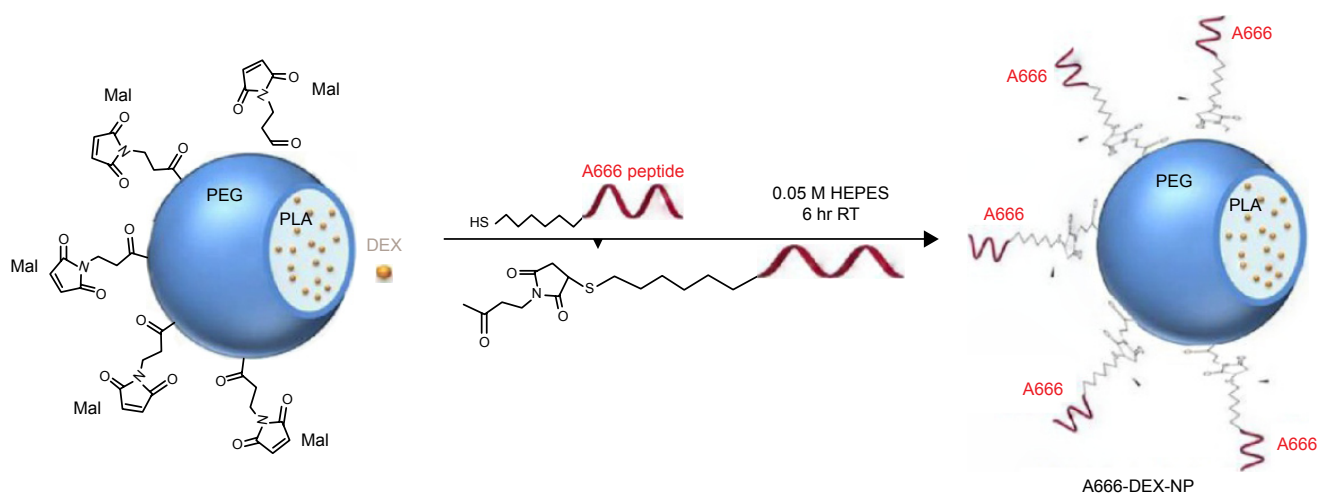

B

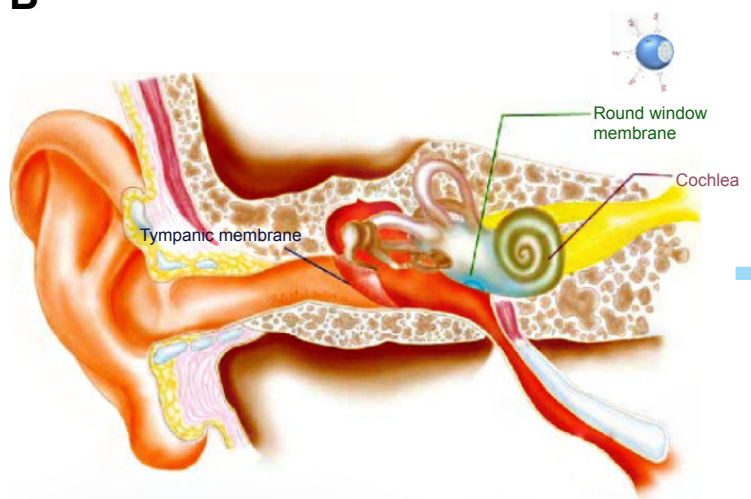

C

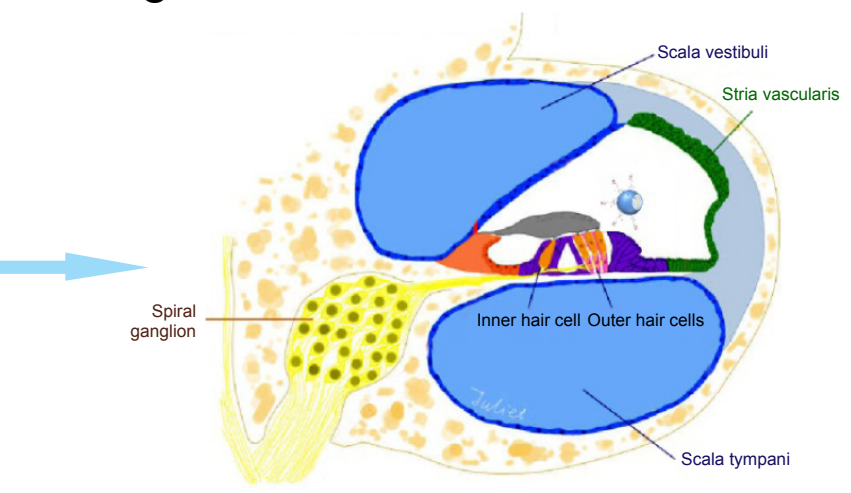

Figure I (A) Preparation of A666-DEX-NP. (B) RWM drug administration. (C) Outer hair cells (OHCs) targeting.

Abbreviations: A666-DEX-NP, A666 peptide-conjugated NP loaded with DEX; DEX, dexamethasone; Mal, maleimide; NP, nanoparticle; OHCs, outer hair cells; PEG, poly(ethylene glycol); PLA, poly(lactide); RT, room temperature; RWM, round window membrane. 
peptide can be calculated as the square root of the mean area occupied by each A666 peptide on the NP surface.

Moreover, lyophilized A666-NP were utilized for X-ray photoelectron spectroscopy (XPS). The XPS analysis was processed on an RBD147 upgraded PHI 5000 C ESCA system (Perkin Elmer, Waltham, MA, USA) using Mg $\mathrm{K} \alpha \mathrm{X}$-rays (hv 1,253.6 eV) and an electron take-off angle of $54^{\circ}$.

\section{Characterization of A666-DEX-NP}

The morphology of A666-DEX-NP was scanned with transmission electron microscope (TEM; Hitachi, Tokyo, Japan). A666-DEX-NP were negatively stained with sodium phosphotungstate solution and scanned under TEM. The average particle size and zeta potential were measured by dynamic light scattering (DLS) detector (Particle Sizing Systems, Santa Barbara, CA, USA). Encapsulation efficiency (EE\%) and drug loading (DL\%) of DEX were conducted using high-performance liquid chromatography-mass spectrometry (HPLC-MS), as described previously. ${ }^{23}$

DEX release from the A666-DEX-NP was performed under artificial perilymph $(\mathrm{pH} 7.4)$ at $37^{\circ} \mathrm{C}$ in a water bath. The artificial perilymph consisted of $137 \mathrm{mM} \mathrm{NaCl}, 5 \mathrm{mM}$ $\mathrm{KCl}, 2 \mathrm{mM} \mathrm{CaCl}_{2}$, and $1 \mathrm{mM} \mathrm{NaHCO}_{3}$ in aqueous solution as a reference. Briefly, a specific amount of A666-DEX-NP was added into $5 \mathrm{~mL}$ artificial perilymph. Then, the solution was placed in the shaking water bath at $37^{\circ} \mathrm{C}$ at $110 \mathrm{rpm}$. Subsequently, the NPs were centrifuged at $11,000 \mathrm{rpm}$ for 30 minutes and replaced with fresh medium. The DEX concentration in the artificial perilymph was measured by HPLC-MS.

\section{Cellular experiments}

\section{Cellular uptake of coumarin 6-labeled A666 conjugated NP}

HEI-OC1 cells were seeded on the VWR ${ }^{\circledR}$ Micro Cover Glasses (VWR, Radnor, PA, USA) at a density of $1.0 \times 10^{4}$ cells/well in 12-well plates. When the cells reached about $80 \%$ confluency, the medium containing A666-coumarin 6-NP $(0.2 \mathrm{mg} / \mathrm{mL}, 500 \mu \mathrm{L})$ was added and incubated over different time points: $0.5,2,5$, and 24 hours. After medium containing NP was removed and the wells washed 3 times with cold phosphate-buffered saline (PBS), 4\% paraformaldehyde (PFA) was added to fix the cells for 20 minutes. Then, the nuclei were stained with DAPI (Sigma, St Louis, MO, USA) for 30 seconds, and the cells were observed under confocal laser scanning microscopy for myosin $7 \mathrm{a}$ (rhodamine; excitation wavelength (Ex): $543 \mathrm{~nm}$, emission wavelength (Em): 565-615 nm) and coumarin 6 (Ex: $488 \mathrm{~nm}$, Em: $505-550 \mathrm{~nm})$.

\section{Cell viability assay of A666-DEX-NP}

CCK-8 was used to evaluate the cytoprotective effect of A666-DEX-NP against CDDP. Briefly, HEI-OC1 cells were seeded in $96-$ well plates at a density of $5.0 \times 10^{3}$ cells/well. After the cells reached about $80 \%$ confluency, the medium was replaced with DEX, DEX-NP, or A666-DEX-NP in culture medium without FBS at DEX concentrations of 2.5, 5, 10, 20, 40, or $80 \mathrm{ng} / \mathrm{mL}$. After 4 hours, $30 \mu \mathrm{M}$ CDDP, in culture medium without FBS, was added to each well. Medium with only saline was used as the control. Blank NP and A666-NP were adjusted to the same concentrations in all cases with NP. After 24 hours incubation, $10 \mu \mathrm{L}$ of CCK-8 solution was added to the cells, incubated for another 2 hours, and the absorbance measured at $450 \mathrm{~nm}$ on a microplate reader (Model 550; Bio-Rad, Hercules, CA, USA).

\section{Cellular apoptosis study}

TUNEL assay was used to quantitatively determine the apoptotic cells pretreated with A666-DEX-NP. Briefly, HEI-OC1 cells were seeded on glass cover slips in 24-well culture plates containing growth medium. After the monolayers achieved $80 \%$ confluence, the medium was replaced with DEX, DEX-NP, or A666-DEX-NP at the same DEX concentration of $80 \mathrm{ng} / \mathrm{mL}$. After 4 hours, the culture medium was replaced with medium containing $30 \mu \mathrm{M}$ CDDP. The medium with saline alone was used as the control. After $24 \mathrm{~h}$ incubation, the cells were washed with cold PBS, fixed in 4\% PFA. Next, the cover glasses were washed in PBS and incubated with the TUNEL reaction mixture for $1 \mathrm{~h}$ at $37^{\circ} \mathrm{C}$. After washing, the slides were incubated with Hoechst 33342 for $30 \mathrm{~min}$ at room temperature. Apoptotic cells were detected in situ utilizing the TUNEL assay (TUNEL POD kit, Roche, Germany). The stained cells were observed under LSM-510 CLSM using an excitation wavelength in the range of 450-550 $\mathrm{nm}$ and a detection wavelength in the range of 515-565 nm. TUNEL positive cells were counted under a $200 \times$ magnification field in triplicate.

\section{Estimation of intracellular ROS levels}

The intracellular levels of ROS were measured using the cell permeable reagent $2^{\prime}, 7^{\prime}$-dichlorofluorescein diacetate (DCFDA; Abcam, Cambridge, MA, USA), a fluorogenic dye that measures hydroxyl, peroxyl, and other ROS activity within the cells. About $5.0 \times 10^{5}$ cells/well were seeded in 6-well plates. After 24 hours incubation, the medium was replaced by saline, DEX, DEX-NP, or A666-DEX-NP at an equivalent DEX concentration of $80 \mathrm{ng} / \mathrm{mL}$. After 4 hours, 
$20 \mathrm{mM}$ DCFDA diluted in serum-free medium was added for 45 minutes at $33^{\circ} \mathrm{C}$. Then, the medium was replaced with $30 \mu \mathrm{M}$ CDDP for another 24 hours. The oxidative burst was analyzed on a fluorescent plate reader with maximum excitation and emission spectra of 495 and $529 \mathrm{~nm}$, respectively.

\section{Cleaved caspase- 3 and $\mathrm{Bcl}-2$ level}

HEI-OC1 cells were seeded in 6-well culture plates at a density of $5.0 \times 10^{5}$ cells/well. After cells reached $80 \%$ confluency, the medium was replaced with saline, DEX, DEX-NP, or A666DEX-NP at the same DEX concentration of $80 \mathrm{ng} / \mathrm{mL}$. After 4 hours incubation, the culture medium containing $30 \mu \mathrm{M}$ CDDP was added to replace the original medium. After 24 hours incubation, the cells were harvested for Western blot.

Briefly, the total lysates of the cells were prepared in lysis buffer. The lysates were centrifuged for 25 minutes at $13,000 \times g$ at $4^{\circ} \mathrm{C}$, and the supernatant was collected. Total protein concentrations were determined using the BCA Protein Assay Kit (Beyotime, Beijing, People's Republic of China). An equivalent of $30 \mu \mathrm{g}$ protein was resolved on $8 \%-12 \%$ SDS-PAGE gels. The following antibodies were used for immunoblot analysis: cleaved caspase-3 (Cell Signaling Technology, Danvers, MA, USA), Bcl-2 (Cell Signaling Technology), and $\beta$-actin (Beyotime) at dilutions ranging from 1:500 to $1: 1,000$. The membranes were developed using an ECL detection kit (Beyotime) and exposed to X-ray film.

\section{Animal experiments OHCs targeting of A666-coumarin 6-NP}

RWM drug administration was performed as previously described. ${ }^{23}$ For in vivo distribution, a total of 6 guinea pigs were randomly assigned to 2 groups for RWM administration of A666coumarin 6-NP and coumarin 6-NP ( $\mathrm{n}=3$, each group). After 2 hours, all guinea pigs were sacrificed via cervical dislocation. Cochleae, harvested from guinea pigs, were fixed in 4\% PFA in PBS for 2 hours, followed by decalcified in 0.12 MEDTA in PBS for 14 days at room temperature. Tissue specimens were cut into $10 \mu \mathrm{m}$ thick sections. Mid-modiolus sections from the cochlea of each animal were used for histological analysis. Myosin $7 \mathrm{a}$ was used to stain the hair cells, while DAPI stained the nuclei. The specimens were observed under CLSM for myosin 7a (rhodamine; Ex: $543 \mathrm{~nm}$, Em: 565-615 nm) and coumarin 6 (Ex: $488 \mathrm{~nm}, \mathrm{Em}: 505-550 \mathrm{~nm}$ ).

\section{In vivo release of A666-DEX-NP}

RWM administration of DEX, DEX-NP, or A666-DEX-NP was effectuated at the DEX concentration of $120 \mu \mathrm{g} / \mathrm{mL}$ ( $\mathrm{n}=5$ for each time point). Approximately $5-7 \mu \mathrm{L}$ perilymph was collected at 1, 3, 6, 12, 24, and 48 hours after drug administration. The perilymph was collected at each time point as previously described. ${ }^{23} \mathrm{DEX}$ concentration in the perilymph was quantified with HPLC-MS as previously described. ${ }^{23}$

\section{A666-DEX-NP preserve hearing loss against CDDP}

The CDDP-induced hearing loss model was obtained as previously described. ${ }^{23}$ The guinea pigs were randomly assigned into 5 groups, with $\mathrm{n}=10 \mathrm{mice} /$ group. 1) CDDP, 2) $\mathrm{CDDP}+\mathrm{A} 666,3) \mathrm{CDDP}+\mathrm{DEX}$, 4) CDDP + DEX-NP, and 5) CDDP + A666-DEX-NP. Briefly, a dose of $12 \mathrm{mg} / \mathrm{kg}$ of CDDP was administered by intraperitoneal injection. DEX $(120 \mu \mathrm{g} / \mathrm{mL}, 5 \mu \mathrm{L})$, DEX-NP $(120 \mu \mathrm{g} / \mathrm{mL}, 5 \mu \mathrm{L})$, and A666-DEX-NP $(120 \mu \mathrm{g} / \mathrm{mL}, 5 \mu \mathrm{L})$ were administered onto the RWM 1 hour before CDDP injection.

The auditory function was measured by auditory brainstem response $(\mathrm{ABR})$ recording. The $\mathrm{ABR}$ thresholds were measured at frequencies of $4,8,16$, and $24 \mathrm{kHz} 1$ day before drug administration and 3 days after, as previously reported. ${ }^{23}$

After the 3-day ABR measurements postdrug administration, the animals were anesthetized with lethal doses of $1 \%$ pentobarbital sodium and subjected to intracochlear perfusion with 4\% PFA in PBS. The temporal bones were decalcified in $0.12 \mathrm{M}$ EDTA in PBS. The hair cell damage was evaluated in whole mounts from groups of guinea pigs treated with different formulation groups. Myosin $7 \mathrm{a}(1: 200)$ was used to identify cochlear hair cells. These whole mounts were examined using a Leica TCS SPE confocal microscope (Leica Microsystems, Wetzlar, Germany). The number of remaining $\mathrm{OHCs}$ was counted in a $1 \mathrm{~mm}$ long strip at the region, approximately $7-10 \mathrm{~mm}$ from the apex.

\section{Statistical analysis}

We used GraphPad Prism 5.0 software (GraphPad Software, La Jolla, CA, USA) for the statistical analysis. The differences between 2 groups were compared using Student's $t$-test, and differences between more than 2 groups were assessed using two-way analysis of variance, followed by Tukey's multiple comparison tests. $P$-values $<0.05$ were considered as statistically significant.

\section{Results}

\section{Characterization of A666-DEX-NP}

A666 peptides were successfully decorated on the surface of NP by confirming the conjugation efficiency of A666 and XPS analysis. The A666 conjugation efficiency was $26.12 \% \pm 3.97 \%$ (Figure 2I). The percentage of sulfur can only be related to the thiolated A666 peptides onto the surface of A666-NP (0.4\%) (Figure $2 \mathrm{G}$ and $\mathrm{H}$ ). 

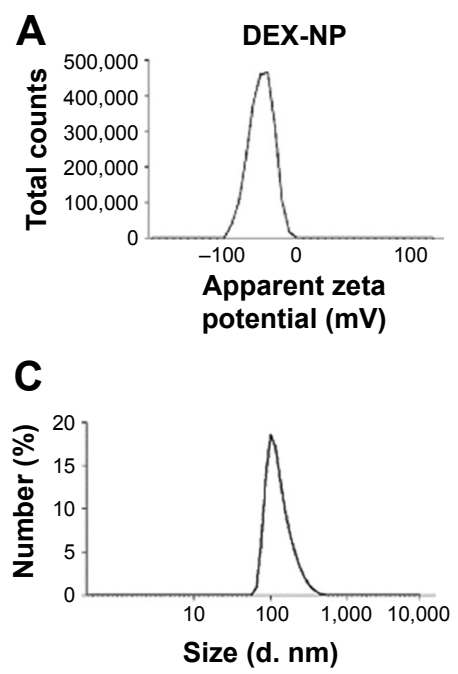

E
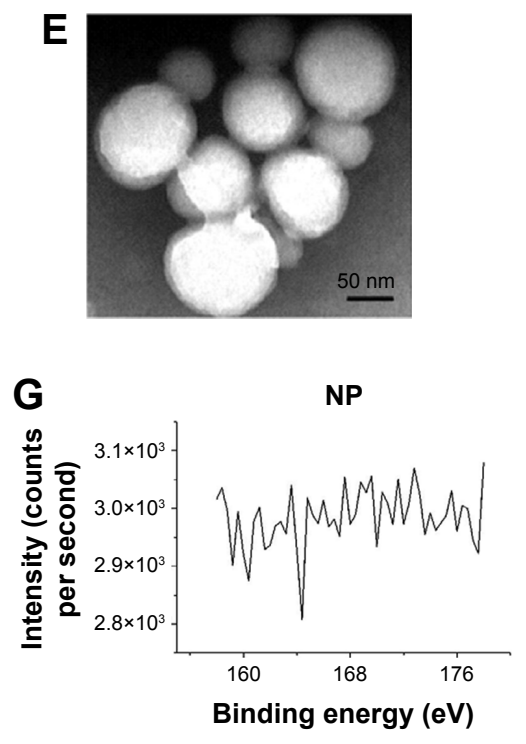
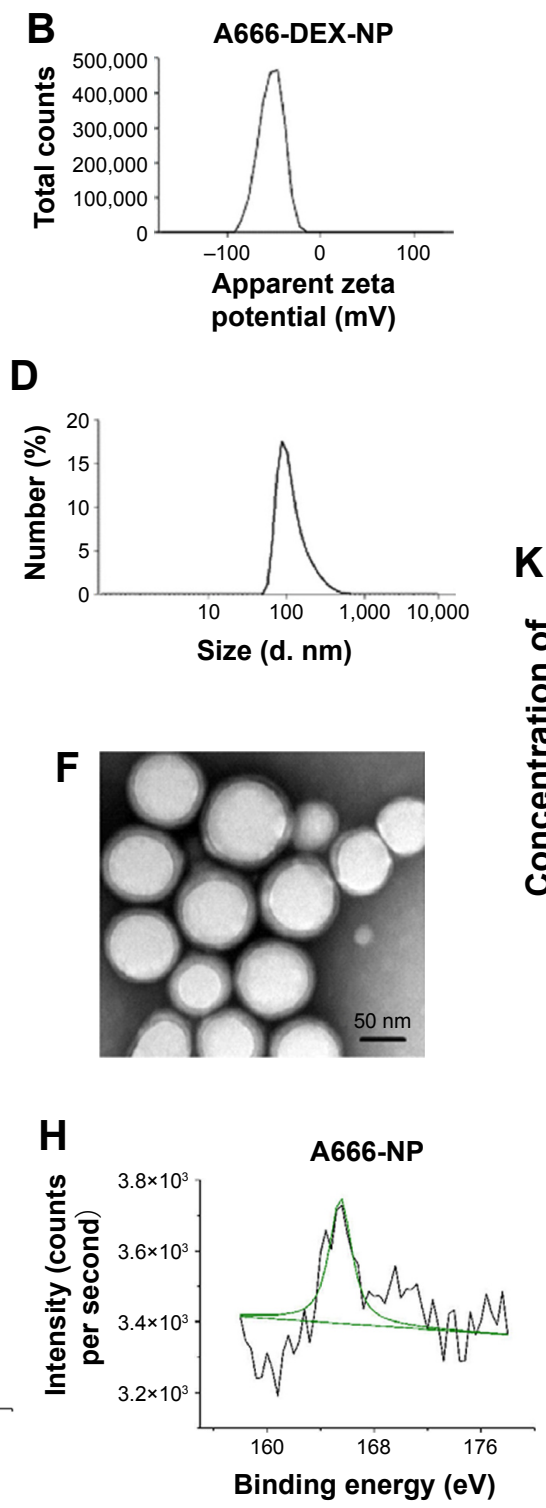
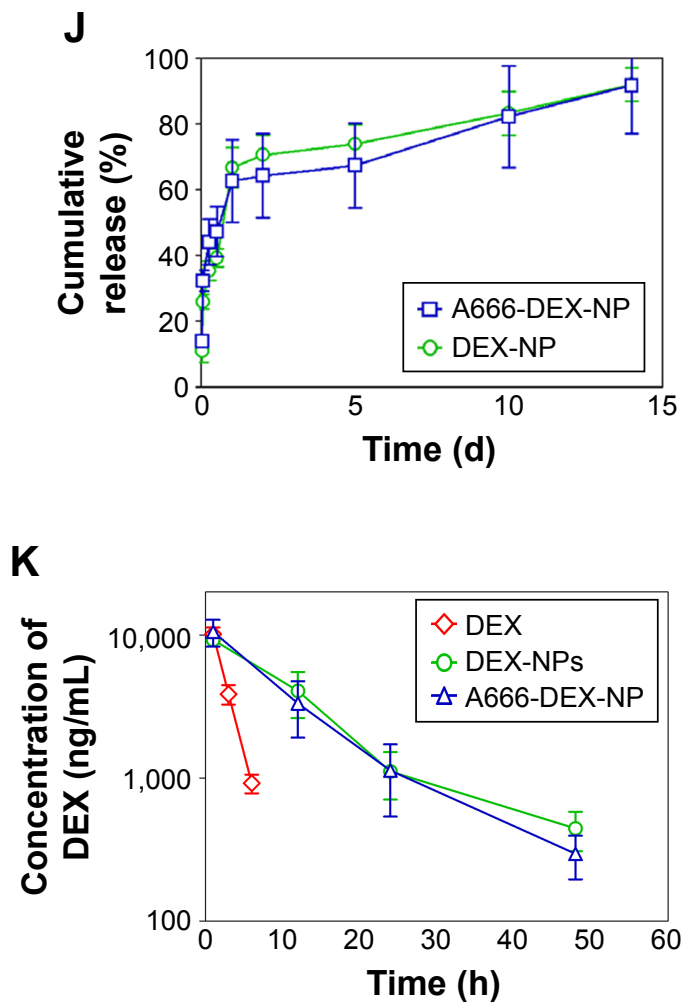

Figure 2 Characterization of A666-conjugated dexamethasone-loaded nanoparticles (A666-DEX-NP).

Notes: Zeta potential of DEX-NP (A) and A666-DEX-NP (B). Particle size and size distribution of DEX-NP (C) and A666-DEX-NP (D). TEM images of DEX-NP (E) and A666-DEX-NP (F). Bar, $50 \mathrm{~nm}$. XPS assay of NP (G) and A666-NP (H). (I) Characteristics of DEX-NP and A666-DEX-NP. S represents A666 surface density. D represents mean distance between neighboring PEG chains which linked to A666 peptide. (J) In vitro release profile of DEX from DEX-NP and A666-DEX-NP in artificial perilymph ( $\mathrm{pH}$ 7.4). (K) In vivo release profiles of DEX in perilymph after RWM administration of DEX, DEX-NP, and A666-DEX-NP ( $n=5$ ). Values are expressed as mean \pm SEM. Abbreviations: A666-DEX-NP, A666 peptide-conjugated NPs loaded with DEX; CE, conjugation efficiency; DEX, dexamethasone; DL, drug loading; DLS, dynamic light scattering; EE, encapsulation efficiency; N/A, not applicable; NP, nanoparticle; RWM, round window membrane; SEM, standard error of the mean; TEM, transmission electron microscopy; XPS, X-ray photoelectron spectroscopy.

No significant differences were observed in zeta potential, particle size, or the morphologies of A666-DEX-NP and DEX-NP (Figure 2A-F). The zeta potential of DEX-NP and A666-DEX-NP was approximately $-30 \mathrm{mV}$. The DLS assay demonstrated that the size of A666-DEX-NP fits a Gaussian distribution and the number-based particle size was $157.80 \pm 14.33 \mathrm{~nm}$, much larger than that observed with TEM. The A666-DEX-NP were uniformly $82.84 \pm 4.52 \mathrm{~nm}$ in size as assessed by the TEM images. The representative TEM micrograph showed that DEX-NP and A666-DEX-NP were uniformly dispersed in water and exhibited spherical shape without aggregation or adhesion (Figure 2E and F). The differences between the 2 NPs in EE and DL were relatively small, suggesting that no significant amount of DEX leaked during the conjugation of DEX-NP with A666.

\section{In vitro release and in vivo release study}

As shown in Figure 2J, we observed an initial burst release followed by a sustained release in the artificial perilymph (pH 7.4). Approximately, $66.75 \%$ DEX from DEX-NP and $62.63 \%$ from A666-DEX-NP were released in the artificial perilymph (pH 7.4) on the first day, and then, $25.14 \%$ and 
$29.03 \%$ were released over the next 13 days, respectively. No statistically significant difference was observed between both DEX-NP and A666-DEX-NP in release profiles, thereby indicating that A666 peptide decoration on the surface of DEX-NP did not alter the release profile of DEX.

The concentration-time courses for DEX, DEX-NP, and A666-DEX-NP after RWM administration are shown in Figure 2K. DEX was removed rapidly from the perilymph within 6 hours after RWM administration. However, DEX-NP and A666-DEX-NP exhibited a remarkably delayed perilymph clearance. DEX concentrations in the cochleae of animals treated with DEX-NP and A666DEX-NP were 444.70 \pm 303.57 and $295.43 \pm 100.04 \mathrm{ng} / \mathrm{mL}$, respectively, at 48 hours. Moreover, no significant difference was observed in the concentration-time curve between DEX-NP and A666-DEX-NP $(P>0.05)$. This result indicated that decoration with A666 peptide on the DEX-NP surface does not influence the in vivo long circulating time of the NPs.

\section{Cellular experiments} Internalization of A666-coumarin 6-NP

A666-coumarin 6-NP were used to monitor the cellular internalization process when added to HEI-OC1 cell culture. The HEI-OC1 cells were exposed to coumarin 6-NP or A666coumarin 6-NP over 2 hours. As shown in Figure 3, the uptake by HEI-OC1 cells of A666-coumarin 6-NP was significantly higher than that of coumarin 6-NP at 5 and 24 hours after incubation, suggesting that A666 peptides decorated on the surface of the NP facilitated the internalization of NP by HEIOC1 $(P<0.05)$. The analysis of the internalization of A666coumarin 6-NP revealed that coumarin 6 (green) and prestin (red) were merged in the cells (yellow), further proving that the internalization of A666-coumarin 6-NP was mainly mediated by the interaction of A666 and prestin which was specifically overexpressed in the plasma of HEI-OC1 cells (Figure 3).

\section{Cell viability assay}

In vitro cell viability of HEI-OC1 cells treated with DEX, DEX-NP, A666-DEX-NP, NP, and A666-NP was examined after 24 hours incubation (Figure 4). The results showed that $\mathrm{NP}$ and A666-NP were noncytotoxic at any tested concentrations $(2.5,5,10,20,40,80 \mathrm{ng} / \mathrm{mL})$ (Figure 4A). In addition, the exposure to $2.5,5,10,20,40$, or $80 \mathrm{ng} / \mathrm{mL}$ of $\mathrm{DEX}$, DEX-NP, or A666-DEX-NP for 24 hours did not exhibit any obvious cytotoxicity to HEI-OC1 cells (Figure 4B).

CDDP exhibited reduced cell viability in a dosedependent manner after exposure to HEI-OC1 for 24 hours (Figure 4C). Half-maximal inhibitory concentration of
CDDP was calculated as $30 \mu \mathrm{M}$. Thus, $30 \mu \mathrm{M}$ CDDP was used in subsequent experiments. When pretreated with 2.5 , 5, 10, 20, 40, or $80 \mathrm{ng} / \mathrm{mL}$ DEX and DEX-NP for 4 hours followed by treatment with $30 \mu \mathrm{M}$ CDDP for 24 hours, the cell viability of HEI-OC1 cells did not reveal any protective effect against CDDP $(P>0.05$ compared to CDDP, Figure 4D and E). Conversely, pretreatment with 2.5, 5, 10, 20, 40, or $80 \mathrm{ng} / \mathrm{mL}$ A666-DEX-NP for 4 hours followed by treatment with $30 \mu \mathrm{M}$ CDDP for 24 hours exerted a maximally protective effect against CDDP at concentrations of 20, 40, and $80 \mathrm{ng} / \mathrm{mL}(P<0.05$ as compared to CDDP, Figure 4F). Following exposure to $30 \mu \mathrm{M}$ CDDP for 24 hours, the cell viability was $45.16 \% \pm 7.61 \%$; however, after pretreatment with 20, 40, or $80 \mathrm{ng} / \mathrm{mL}$ A666-DEX-NP for 4 hours, the cell viability was restored to $97.27 \% \pm 10.98 \%, 98.54 \% \pm 11.32 \%$, and $96.37 \% \pm 14.23 \%$, respectively. This suggested that pretreatment with A666-DEX-NP $(20,40$, or $80 \mathrm{ng} / \mathrm{mL})$ dramatically restored the viability of HEI-OC1 cells.

\section{A666-DEX-NP attenuates CDDP-induced apoptosis in $\mathrm{HEI}-\mathrm{OCl}$ cells}

TUNEL staining showed that pretreatment with DEX-NP or A666-DEX-NP decreased apoptosis, while pretreatment with DEX did not affect apoptosis (Figure 5A and B). Furthermore, in contrast to DEX-NP, A666-DEX-NP showed a remarkably greater decrease in apoptosis. CDDP exposure increased the activation of the apoptotic effector, cleaved caspase-3, while A666-DEX-NP pretreatment reduced the levels of cleaved caspase- 3 and instead increased the expression of the antiapoptotic protein, Bcl-2 (Figure 5C-E). These results indicated that A666-DEX-NP significantly inhibited the CDDP-mediated apoptosis.

\section{A666-DEX-NP inhibits CDDP-induced ROS production}

The levels of ROS in the groups treated with CDDP, and in the groups pretreated with DEX, DEX-NP, or A666DEX-NP for 4 hours followed by CDDP exposure were $378.20 \% \pm 60.47 \%, 312.58 \% \pm 49.42 \%, 269.70 \% \pm 26.48 \%$, and $272.09 \% \pm 17.42 \%$, respectively, as compared to those in the control. Thus, the CDDP-induced elevation of ROS was significantly decreased by DEX-NP and A666-DEX-NP pretreatment. $(P<0.001$, Figure 5F).

\section{Animal experiments}

In vivo OHCs targeting of A666-coumarin 6-NP

The selectivity of A666-NP toward OHCs was confirmed in guinea pigs. Following 2 hours of RWM administration, the con- 
A
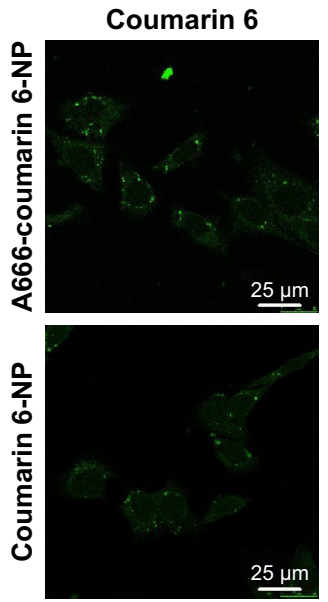

Prestin
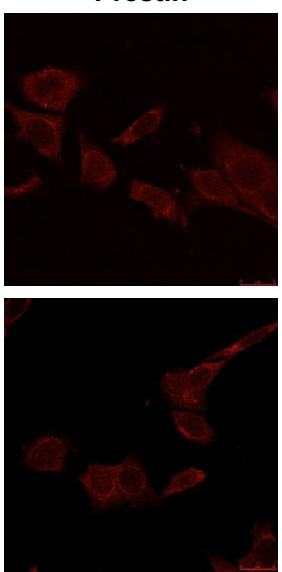

DAPI
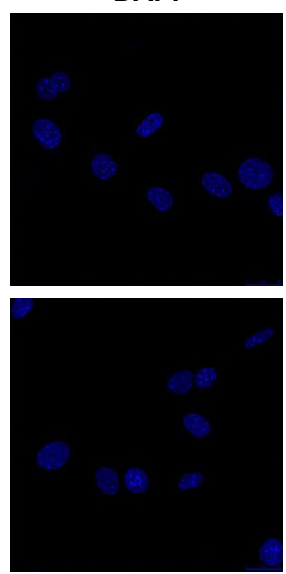

Merge
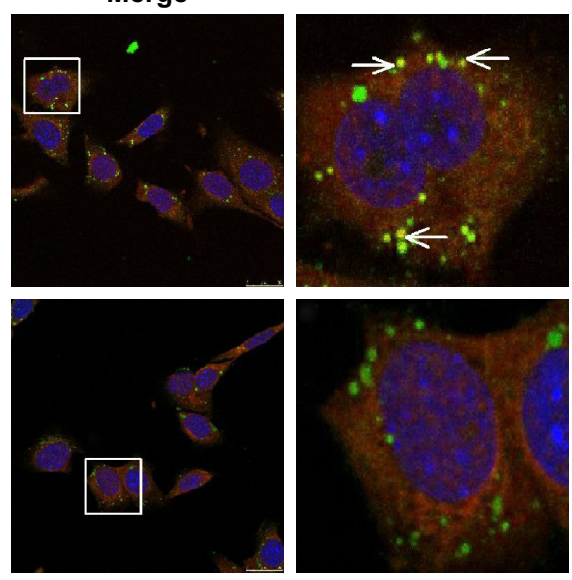

B

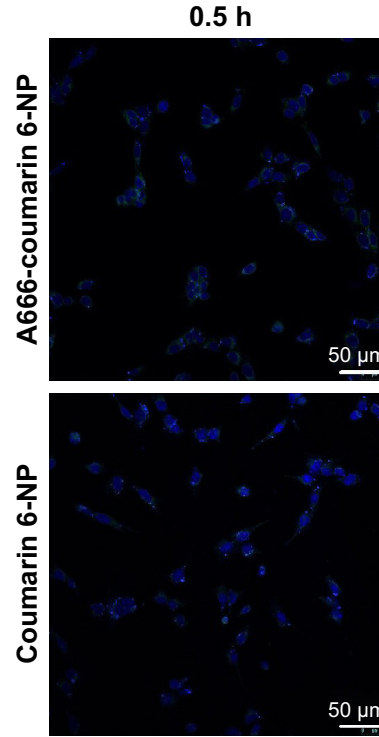

$2 \mathrm{~h}$
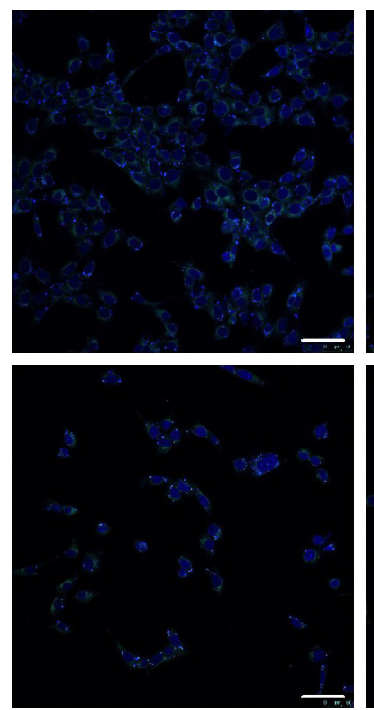

$5 \mathrm{~h}$
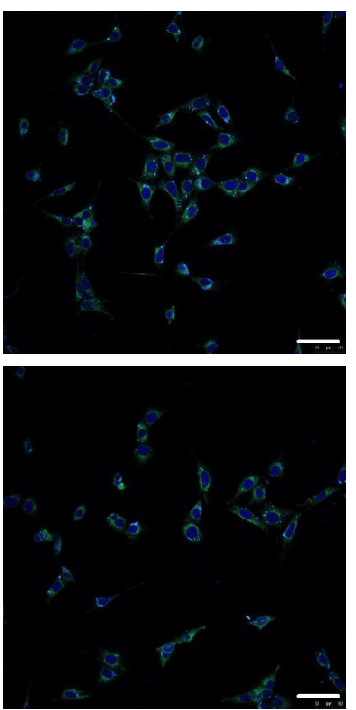
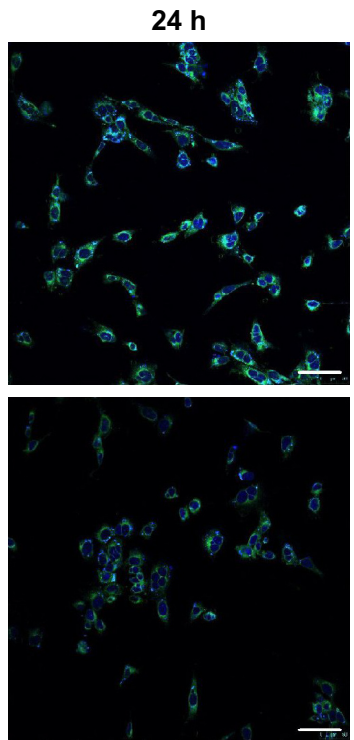

C

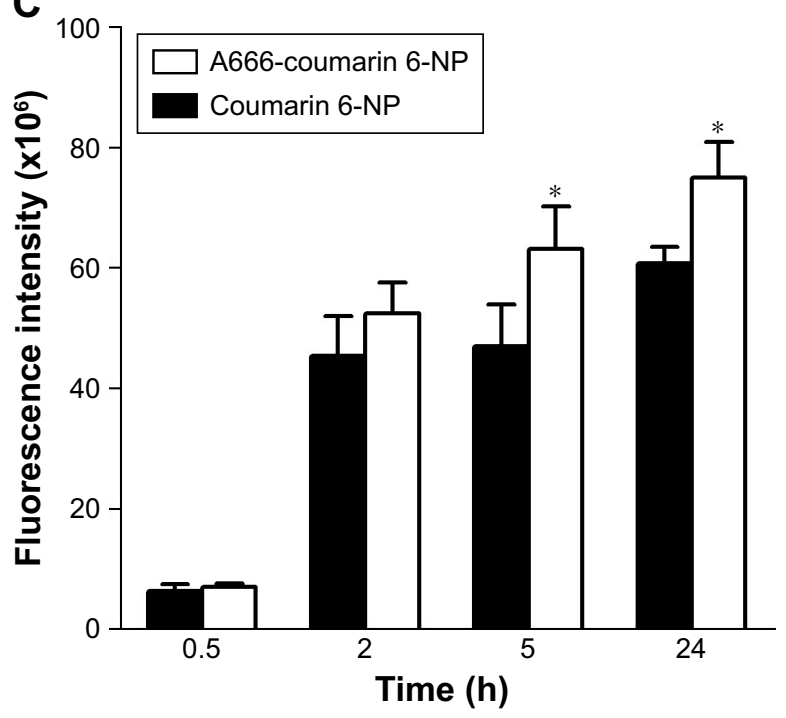

Figure 3 (A) Representative CLSM image of HEl-OCI after $2 \mathrm{~h}$ culture with A666-coumarin 6-NP and coumarin 6-NP. Red indicates prestin staining, green indicates the NPs inside the cells, and blue represents the nuclei stained by DAPI. Arrows, colocalization of prestin and coumarin 6 . Scale=25 $\mu \mathrm{m}$. (B) Representative CLSM images of HEI-OCI after $0.5,2,5$, and $24 \mathrm{~h}$ culture with A666-coumarin 6-NP and coumarin 6-NP. Scale=50 $\mu \mathrm{m}$ (C) Fluorescence intensity in HEI-OCI incubated with A666-coumarin 6-NP and coumarin 6-NP after $0.5,2,5$, and 24 h. $* P<0.05$ as compared to coumarin 6-NP.

Abbreviations: CLSM, confocal laser scanning microscopy; NP, nanoparticle. 

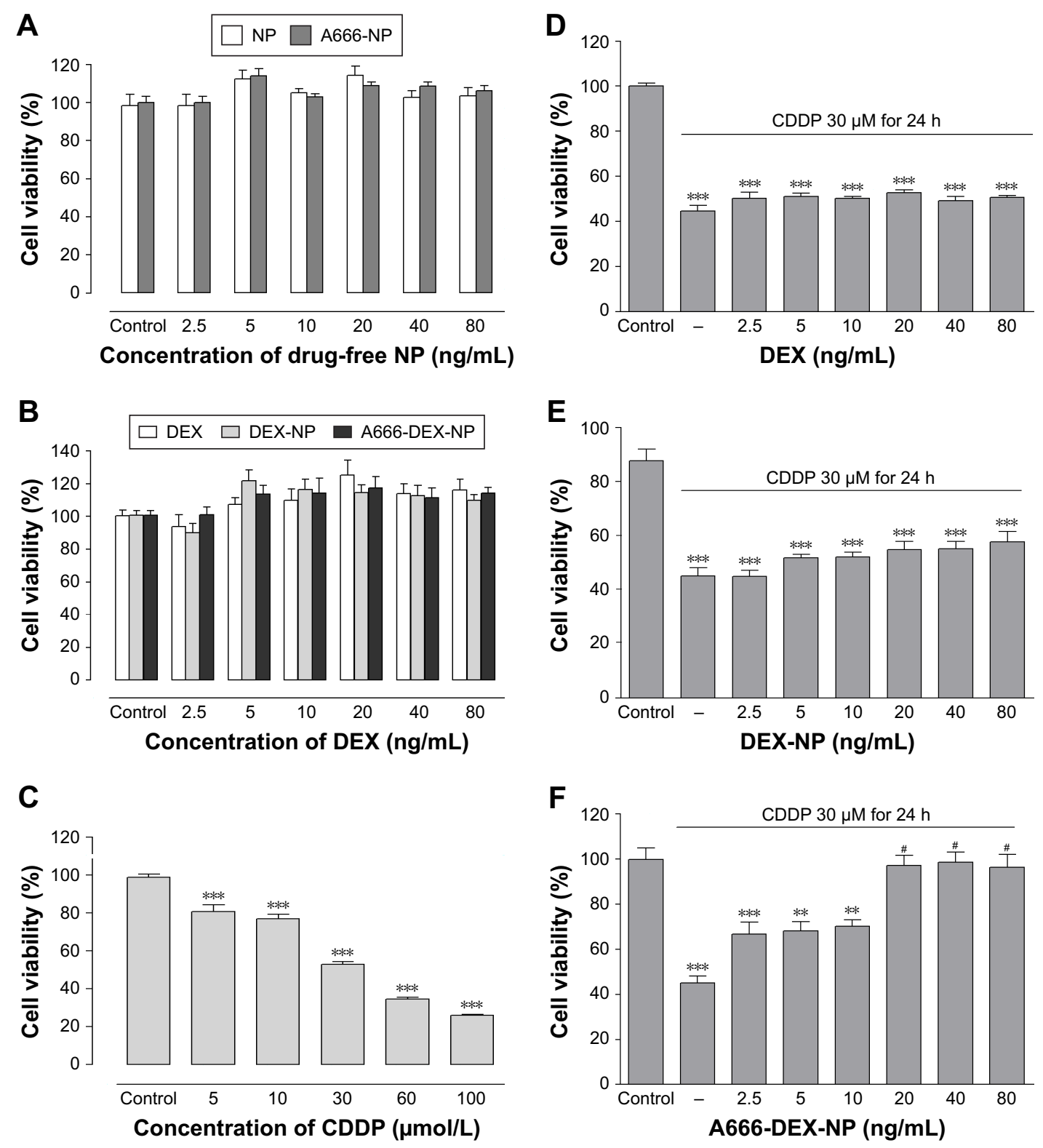

Figure 4 Effect of various DEX formulation, DEX, DEX-NP and A666-DEX-NP, NP and A666-NP on CDDP-mediated decrease in viability of HEI-OCI cells. Notes: (A) Effect of NP and A666-NP on HEI-OCI cells viability at experimental concentrations. (B) Effect of DEX, DEX-NP, and A666-DEX-NP on HEI-OCI cells viability at experimental concentrations. (C) CDDP decreased cell viability is dose dependent (5-100 $\mu \mathrm{mol} / \mathrm{L})$. CDDP (30 $\mu \mathrm{M})$ treatment for $24 \mathrm{~h}$ decreased the cell viability by c.a. $50 \%$. The effect of DEX (D) DEX-NP (E) and A666-DEX-NP (F) on CDDP-mediated decrease in viability of HEl-OCI cells. The values are expressed as mean \pm SEM, $\mathrm{n}=6$. $* * P<0.01$, $* * * P<0.001$ as compared to the control. ${ }^{*}<0.05$ as compared to the CDDP.

Abbreviations: A66-DEX-NP, A666 peptide-conjugated NPs loaded with DEX; CDDP, cisplatin; DEX, dexamethasone; NP, nanoparticle; SEM, standard error of the mean.

focal images showed that most of the A666-modified coumarin 6-NP targeted to the OHCs of guinea pigs and was only sparsely taken up by the other cell types (cells in the stria vascularis, spiral ganglion). Conversely, the coumarin 6-labeled NP without conjugated A666 did not home to the OHCs (Figure 6).

Low-magnification images of the samples showed the overviews of the whole cochlea. For the control group, weak fluorescence was observed in the spiral ganglion cells. For A666-coumarin 6-NP and coumarin 6-NP groups, stronger fluorescence accumulated in ogan of Corti, spiral ganglion, and stria vascularis (Figure 6A).
High-magnification images of the samples incubated with A666-coumarin 6-NP displayed a stronger signal in the $\mathrm{OHCs}$ of the guinea pigs, demonstrating targeted delivery to the cells (Figure 6B).

\section{A666-DEX-NP protect from CDDP-induced hearing loss}

Conventional ABR recording was used to monitor the auditory function after CDDP treatment. As shown in Figure 7, the ABR tests illustrated that A666-DEX-NP partially protected from CDDP-induced hearing loss on day 3 after RWM 
A
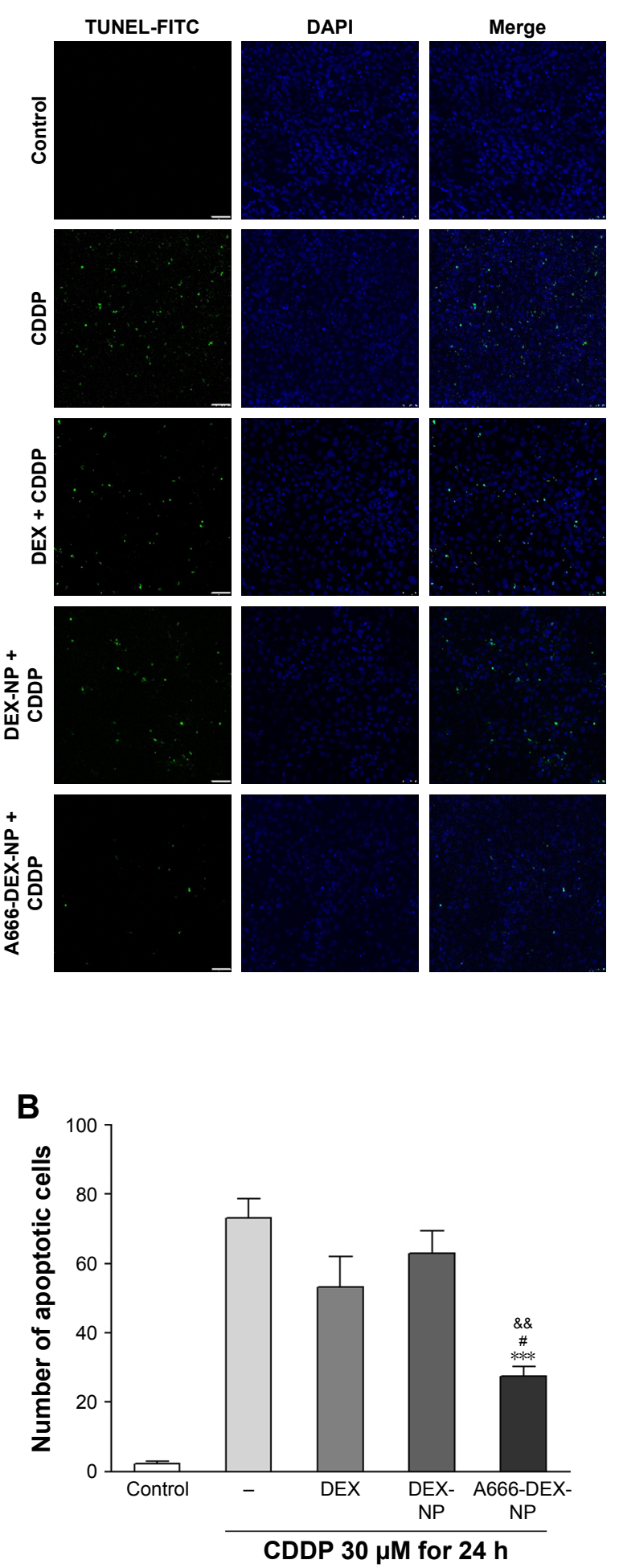

C

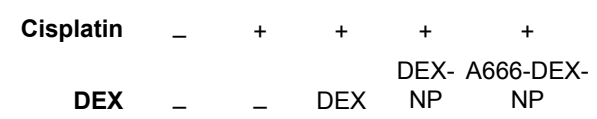

Cleaved caspase-3

(17 kDa)

Bcl-2 (26 kDa)

Actin (42 kDa)

D

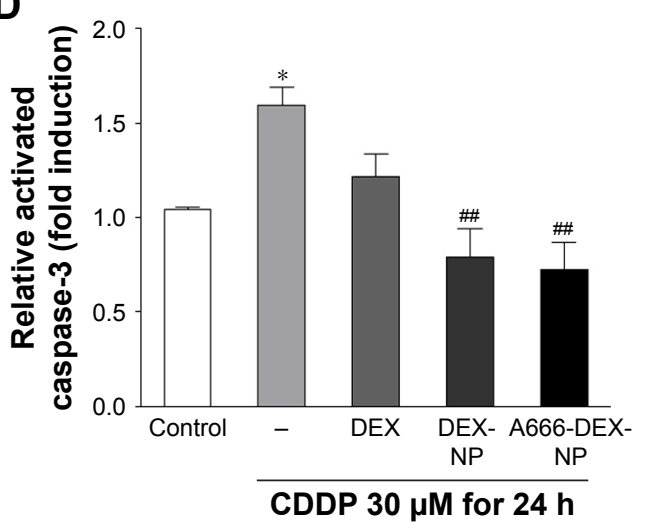

E

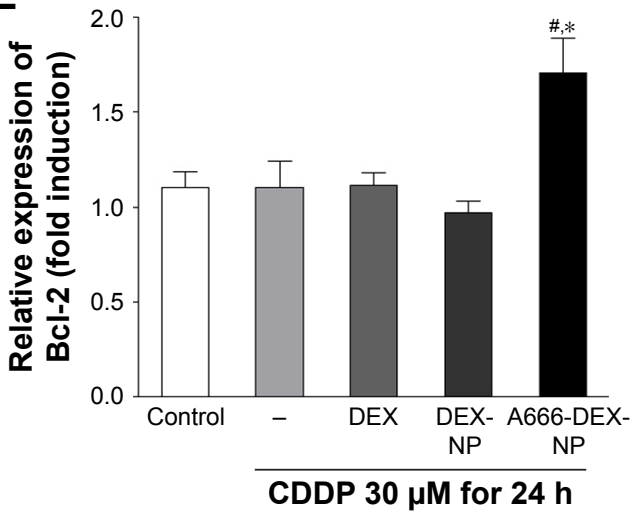

$\mathbf{F}$

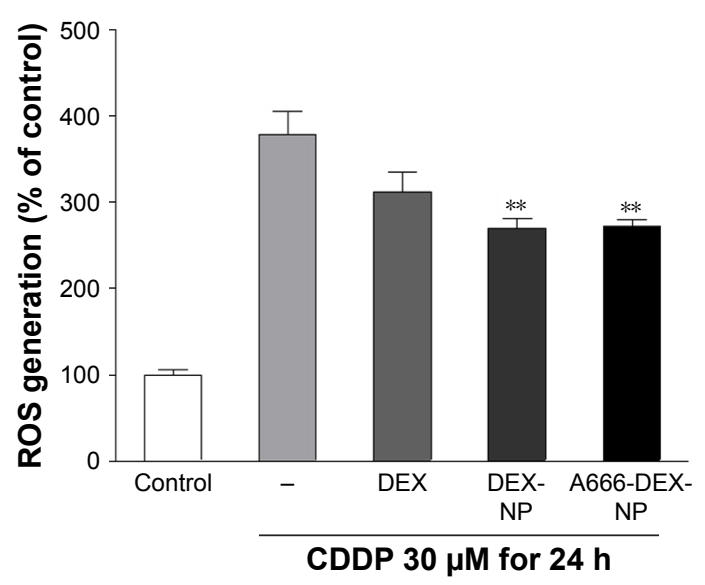

Figure 5 Protective effects of DEX, DEX-NP, and A666-DEX-NP on apoptosis of CDDP-induced HEI-OCI cells.

Notes: HEI-OCl were pretreated with DEX, DEX-NP, and A666-DEX-NP ( $80 \mathrm{ng} / \mathrm{mL})$ for $4 \mathrm{~h}$, followed by CDDP (30 $\mu$ M). (A) A666-DEX-NP decreased the apoptosis of $\mathrm{HEI}-\mathrm{OCl}$ cells. Bar, $50 \mu \mathrm{m}$. (B) Quantification of the number of TUNEL-positive cells. $* * * P<0.00 \mathrm{I}$ as compared to CDDP, \#P<0.05 as compared to CDDP + DEX. \&\&P $<0.01$ as compared to CDDP + DEX-NP. (C-E) A666-DEX-NP pretreatment inhibited the expression of apoptotic effector (cleaved caspase-3) and increased the antiapoptotic protein (Bcl-2) $* P<0.05$ as compared to control. ${ }^{*} P<0.05,{ }^{\# P}<0.01$ as compared to CDDP. (F) ROS production. CDDP significantly increased intracellular ROS levels. Pretreatment with DEX-NP and A666-DEX-NP for $4 \mathrm{~h}$, followed by $30 \mu \mathrm{M}$ CDDP remarkably decreased the production of intracellular ROS. Data are presented as mean \pm SEM of triplicate determinations. **P $<0.0$ I as compared to CDDP. “-" in $x$-axes represents no pretreated drug.

Abbreviations: A666-DEX-NP, A666 peptide-conjugated NPs loaded with DEX; CDDP, cisplatin; DEX, dexamethasone; NP, nanoparticle; ROS, reactive oxygen species; SEM, standard error of the mean. 
A

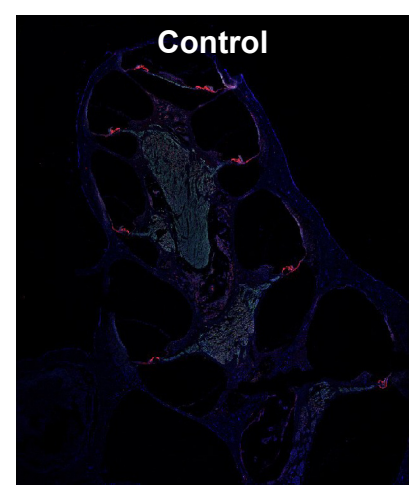

B
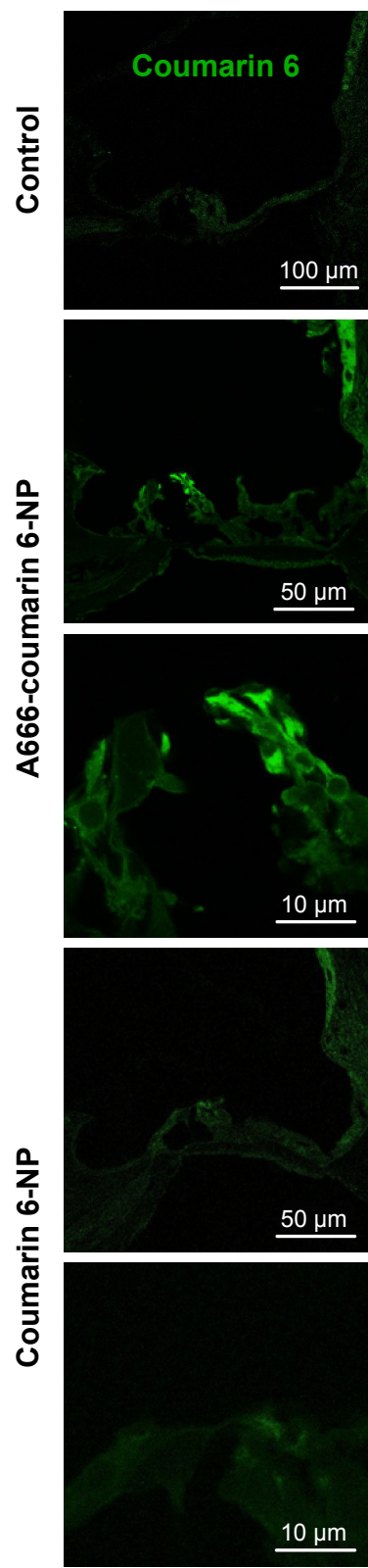

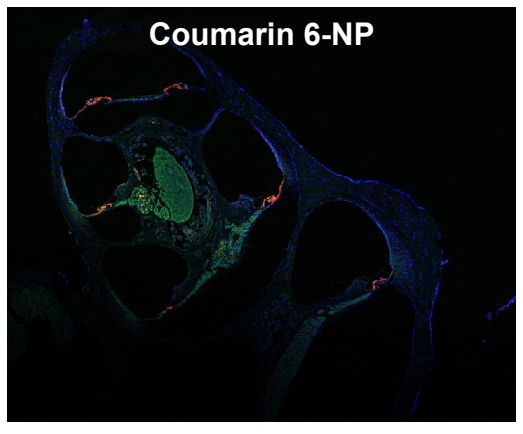

\section{A666-coumarin 6-NP}
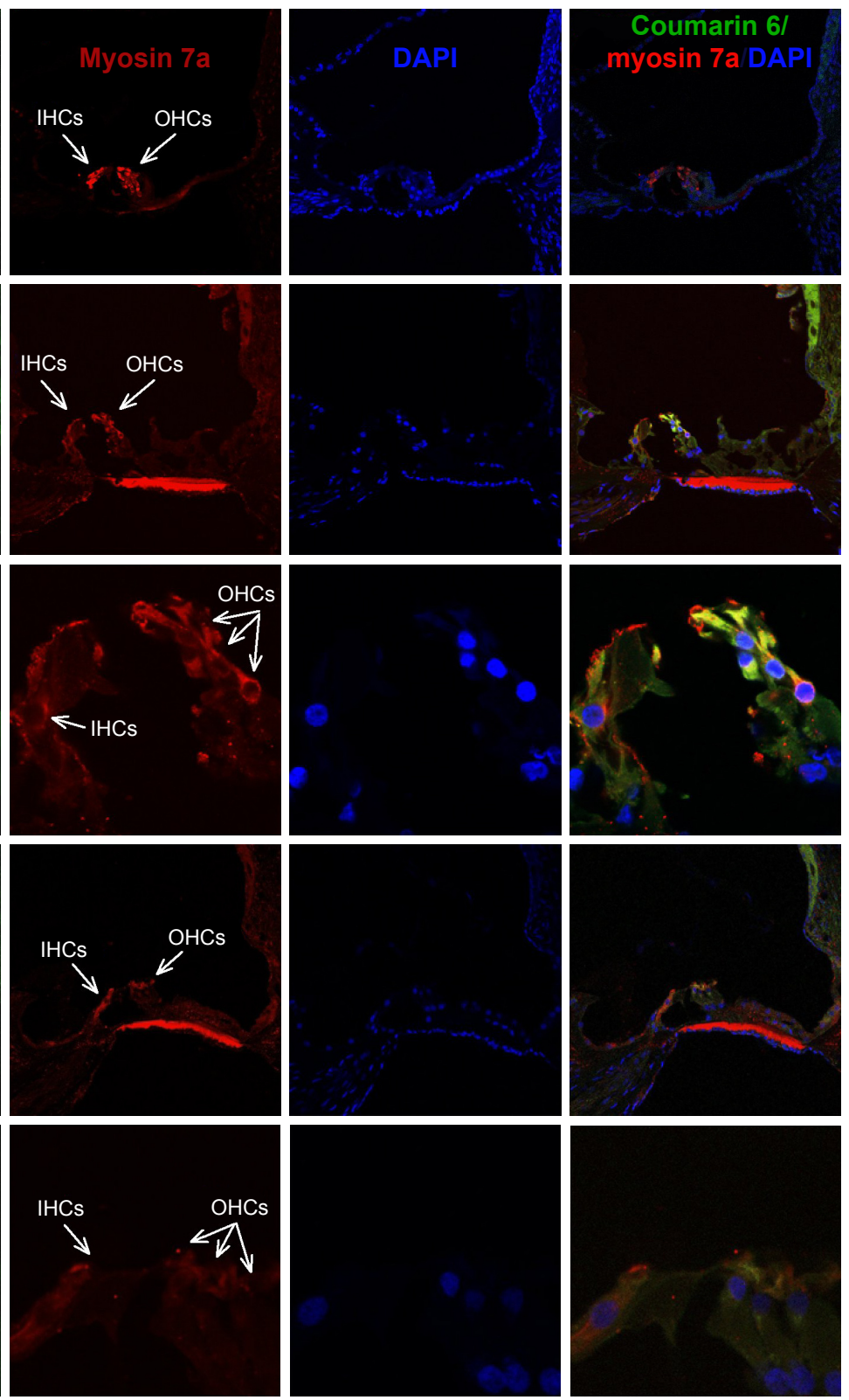

Figure 6 In vivo OHCs targeting of coumarin 6-labeled A666-NP (A666-coumarin 6-NP).

Notes: Three guinea pigs in each group were treated with coumarin 6-NP (green) with or without A666 decoration on round window membrane by a single dose. After 2 h, the animals were sacrificed, and the inner ear was harvested, fixed, and sectioned. The cochleae were immunostained for myosin 7a (OHCs) (red). (A) Whole inner ear of guinea pigs treated with A666-coumarin 6-NP or coumarin 6-NP. (B) The organ of Corti treated with A666-coumarin 6-NP or coumarin 6-NP. Most A666-coumarin 6-NP accumulate on $\mathrm{OHC}$, while coumarin 6-NP showed no $\mathrm{OHC}$ targeting.

Abbreviations: IHC, inner hair cell; NP, nanoparticle; OHC, outer hair cell. 
A

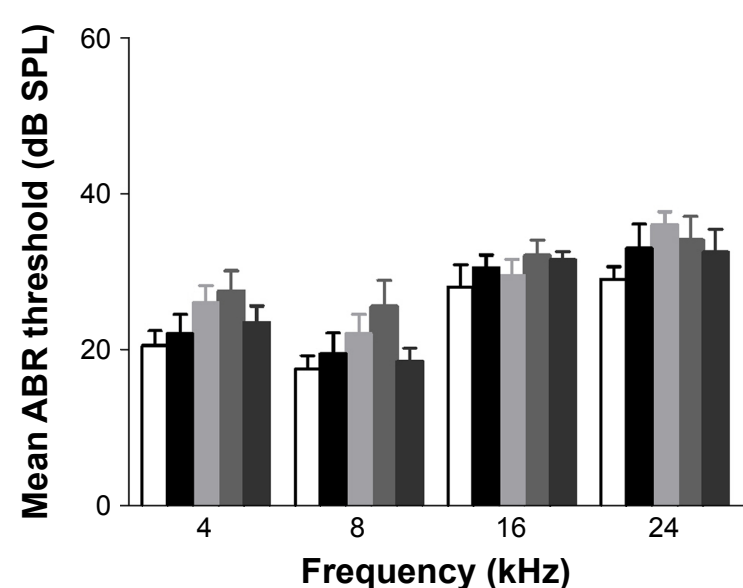

B

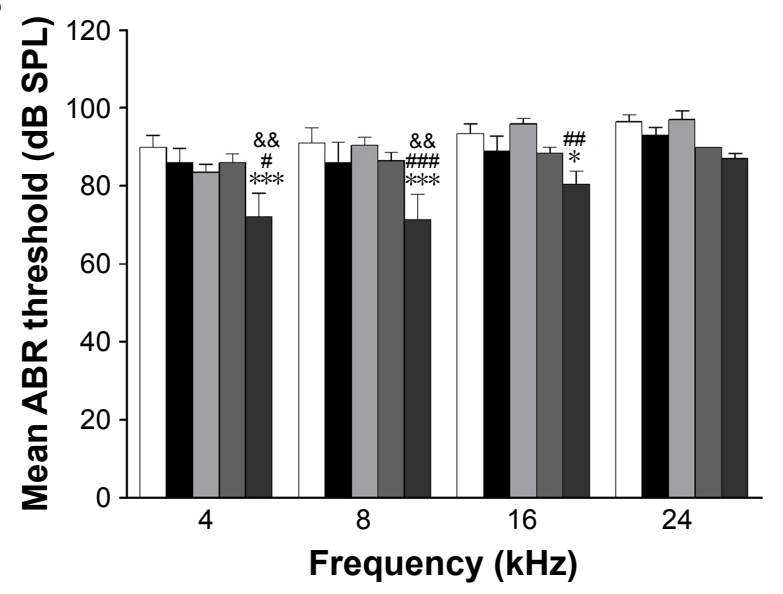

$\square$ CDDP $\square$ CDDP + A666 $\square$ CDDP + DEX $\square$ CDDP + DEX-NP $\square$ CDDP + A666-DEX-NP

C
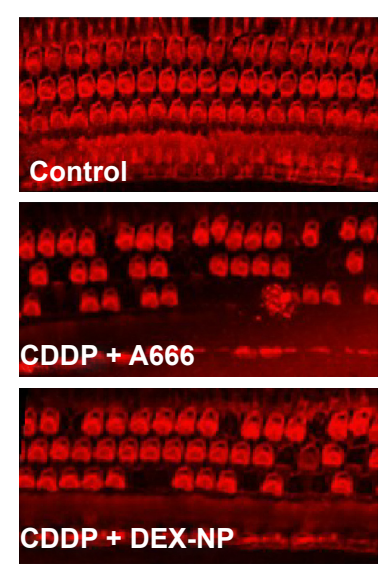
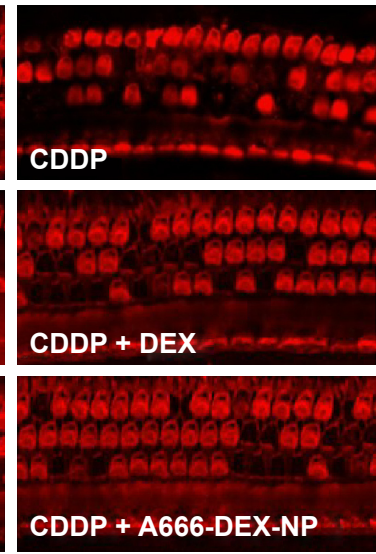

D

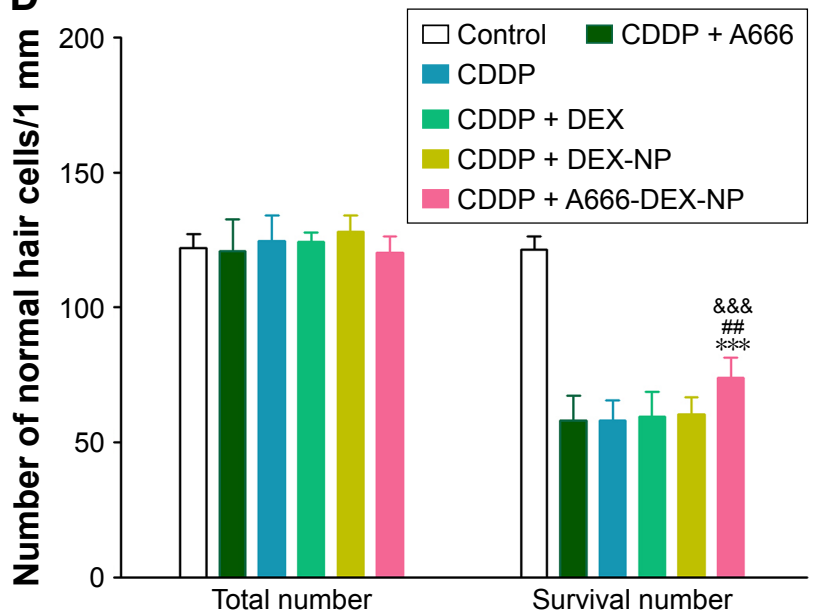

Figure 7 A666-DEX-NP protected from CDDP-induced hearing loss.

Notes: Mean ABR threshold of guinea pigs I d before $(\mathbf{A})$ and $3 \mathrm{~d}$ after (B) RWM administration. The mean ABR threshold is represented as the mean \pm SEM. (C) Rhodaminephalloidin staining of the $60 \%-80 \%$ from the apex of Corti. (D) The number of survived OHCs in I mm length is about $60 \%-80 \%$ from the apex of each cochlea. $* P<0.05$,

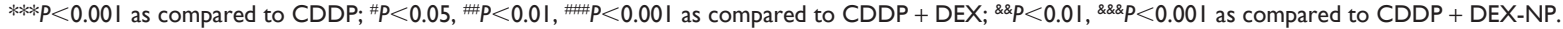

Abbreviations: A666-DEX-NP, A666 peptide-conjugated NPs loaded with DEX; ABR, auditory brainstem response; CDDP, cisplatin; DEX, dexamethasone; NP, nanoparticle; OHCs, outer hair cells; RWM, round window membrane; SEM, standard error of the mean.

administration. Compared to CDDP, CDDP + A666-DEX-NP exhibited a significantly enhanced protective effect at $4 \mathrm{kHz}$ $(P<0.001), 8 \mathrm{kHz}(P<0.001)$, and $16 \mathrm{kHz}(P<0.05)$. However, the CDDP + DEX and CDDP + DEX-NP groups did not show a statistically significant difference in the mean $A B R$ threshold at all the detected frequencies. The CDDP + A666 did not have any protective effect compared to the CDDP group. Furthermore, CDDP + A666-DEX-NP showed a higher preventive effect than CDDP + DEX and CDDP + DEX-NP at 4,8 , and $16 \mathrm{kHz}$ frequencies (Figure $7 \mathrm{~A}$ and $\mathrm{B}$ ).

Furthermore, in order to investigate the ability of A666DEX-NP in protecting these cells, the surviving hair cells were histologically counted. As shown in Figure7C and D, compared to control, the CDDP group exhibited a severe loss of OHCs and abnormalities. On the other hand, the CDDP + A666-DEX-NP rescued the hair cells from CDDP damage, effectively. The quantification of the survival of $\mathrm{OHCs}$ showed consistent results with those of ABR (Figure 7).

\section{Discussion}

Exploring strategies to combat CDDP-induced ototoxicity is yet challenging. The failures of clinical trials of otoprotective agents force scientists to take advantage of nanotechnology to achieve this goal. Among them, DEX benefits from the DDS. Recently, a poloxamer hydrogel OTO-104 containing DEX has been reported to dramatically increase the residence time of DEX in the inner ear and significantly protect against the CDDP-induced ototoxicity. ${ }^{24}$ 
Alternatively, DEX-loaded NPs administered by bullectomy provided notable protection against CDDP-induced ototoxicity. ${ }^{25}$ In our previous study, self-assembled NPs loaded with DEX in guinea pigs showed that high concentrations of DEX were to be achieved in the inner ear to provide significant protection against CDDP-induced ototoxicity as compared to DEX administration. ${ }^{23}$ To further expand the otoprotective range and intensity of NPs loaded with DEX system, we developed a novel OHCs targeting delivery system: A666-decorated NPs.

We designed the amphiphilic polymers, Mal-PEG-PLA and mPEG-PLA. After oil/water emulsion and solvent evaporation, the maleimide groups protruded out of the surface of the NPs (Figure 1). A666 was cationized and thiolated, followed by conjugation through the maleimide function located at the distal end of PEG surrounding the nanoparticle's surface (Figure 1). The high degree of specificity and reactivity of sulfhydryl groups with maleimide moieties to form stable thioether bonds has been exploited extensively for the construction of immobilized antibodies, enzymes, and peptide-conjugated haptens. ${ }^{27}$ In addition, this reaction is widely used for conjugation of cysteinecontaining peptides and proteins. ${ }^{28,29}$ In contrast, the binding of small peptides (such as A666 peptide, which is isolated from 3 rounds of sequential phage display peptide libraries) to their receptors is highly specific with high affinity, and they are generally considered less immunogenic than IgG antibodies. ${ }^{26}$

One of the main issues investigated in this study was whether A666-NP can penetrate the RWM and accumulate to OHCs. A666 peptide decoration on the NPs surface can potentially facilitate the uptake of NP by HEI-OC1 cells. The colocalization of A666-coumarin 6-NP and prestin further demonstrated that the internalization of A666-NP was mainly mediated by the recognition of A666 and prestin specifically overexpressed in HEI-OC1 cells. The current study confirmed that A666-coumarin 6-NP was targeted to the OHCs of guinea pigs within 2 hours of administration. In contrast, coumarin 6-NP did not home to the OHCs. In addition to OHCs, A666-coumarin 6-NP was found in the stria vascularis and spiral ganglion cells. Thus, we speculated that A666-coumarin 6-NP entered the perilymph first, passed through the stria vascularis into the endolymph, and then, penetrated to $\mathrm{OHCs}$, instead of entering the endolymph directly through the basilar membrane after RWM administration.

After optimizing the main process parameters for A666DEX-NPs with respect to their EE and DL, the amount of
A666 linked to the particle surface was determined using a rapid and highly sensitive CBQCA-based fluorescence method. ${ }^{22}$ The results showed that approximately 3,490 A666 molecules were conjugated on the surface of each NP, and the mean distance between 2 neighboring PEG chains linked to an A666 peptide was $4.85 \mathrm{~nm}$. The multivalent array of A666 ligands on the surfaces of the NPs offered stronger binding affinity than prestin, with multivalent linking to the targeted cells, and facilitated the ligandreceptor-mediated internalization of the NPs. The in vitro and in vivo release profiles of drug-loaded NPs represent the major parameters that influence the therapeutic effects. ${ }^{30}$ The decoration of A666 peptide did not change the release rate of the NPs. This can be easily explained because the conjugation reaction occurs with the maleimide groups protruding from the surface of NPs without interfering with the polymer matrix. In artificial perilymph media, DEX displayed a biphasic release pattern, specifically there was an initial fast release and a subsequent slower sustained release. In vivo, DEX-NP and A666-DEX-NP could extend the drug retention of DEX in perilymph from 6 to 48 hours. These results demonstrated that A666-DEX-NPs are efficient in delivering DEX in a sustained manner to the OHCs.

The improved uptake of A666-NP and long-term retention in the perilymph resulted in the anticipated enhanced otoprotective activity with respect to CDDP-induced HEI-OC1 cytotoxicity. Thus, we demonstrated the protective activity of A666-DEX-NP against CDDP-induced hair cell damage. Blank NP and A666-NP, DEX, DEX-NP, and A666-DEX-NP were tested in HEI-OC1 cells in order to test their cytotoxicity; none were found to be cytotoxic at all concentrations of DEX. Compared to DEX and DEX-NP, pretreatment with A666-DEX-NP significantly antagonized the CDDP-induced cytotoxicity. Pretreatment with A666-DEX-NP for 4 hours, followed by CDDP for 24 hours, reversed the HEI-OC 1 cytotoxicity to normal level. Moreover, A666-DEX-NP exhibited a higher protective activity against apoptosis induced by CDDP as compared to DEX and DEX-NP. Thus, we speculated that sustained release of DEX from DEX-NP was timeconsuming if the high cytotoxic protective activity was to be achieved in contrast to A666-DEX-NP; however, DEX might be available to the cells immediately. Compared to DEX-NP, A666 peptide and PEG "corona" endowed A666-DEX-NP with targeting ability and extended the circulation time, and this increased the chance of binding to prestin receptors on $\mathrm{OHCs}$ in the inner ear.

CDDP-induced ototoxicity is usually presented as bilateral irreversible sensorineuronal hearing loss. CDDP interacts 
with cochlear tissues such as OHCs of organ of Corti, induces degeneration of the stria vascularis, and decreases the number of spiral ganglion cell. ${ }^{31} \mathrm{CDDP}$ entry into $\mathrm{OHCs}$ results in cell death, which appears to be primarily caspase dependent. ${ }^{32}$ CDDP-induced apoptosis in the inner ear could be due to the activation of different pathways. Caspase-dependent pathway culminates in the activation of caspase-3, which cleaves several substrates resulting in chromosomal DNA fragmentation and cellular morphologic changes characteristic of apoptosis. ${ }^{33}$ About 80 ng/mL A666-DEX-NP significantly reduced the caspase- 3 activity in cells treated with CDDP over 24 hours. However, DEX and DEX-NP did not show reduced caspase- 3 activity at the same concentration. A recent study demonstrated that the intracochlear perfusion of a caspase-3 inhibitor (z-DEVD-fmk) significantly reduced the ototoxic effects of CDDP. ${ }^{21}$ Therefore, the inhibition of proapoptotic biomarkers such as caspase-3 activity by A666-DEX-NP and the protection against CDDP-induced cytotoxicity in vitro suggested that these formulations might reduce the ototoxicity in an in vivo model.

The present study demonstrated that A666-DEX-NP attenuated the CDDP-induced damage to the OHCs at 4, 8 , and $16 \mathrm{kHz}$ based on ABR and hair cells staining results following RWM administration. This phenomenon might be related to the active targeting of $\mathrm{OHCs}$ and the long, sustained drug release characteristics. In a previous study, we found that DEX-NP statistically protected CDDP-induced hearing loss at 4 and $8 \mathrm{kHz}$ as compared to DEX formulation. ${ }^{23}$ However, the DEX concentration used in our previous study $(10 \mathrm{mg} / \mathrm{mL}, 10 \mu \mathrm{L})$ was distinctly higher than used in the present study $(120 \mu \mathrm{g} / \mathrm{mL}, 10 \mu \mathrm{L})$. This phenomenon further proved that A666-DEX-NP enhanced the protective effect of OHCs targeted by A666 peptide decoration. This active OHCs-targeting property endowed OHCs high drug concentration with A666-DEX-NP. On the other hand, A666-DEX-NP exhibited a slight protective effect on CDDPinduced hearing loss at $16 \mathrm{kHz}$. The enhanced protective effect is expected to be achieved by improved drug concentration loaded in A666-NP.

\section{Highlights}

- A novel outer hair cells (OHCs)-targeted dexamethasone (DEX)-loaded drug delivery system, A666-DEX-NP, was developed successfully.

- OHC-targeting property of A666-NP was confirmed in vitro with HEI-OC1 cell line and in vivo with guinea pigs.

- A666-DEX-NP exhibited a long-sustained release characteristic of the in vitro artificial perilymph (14 days) and in vivo perilymph (48 hours) by round window membrane administration.

- A666-DEX-NP $(20,40$, or $80 \mathrm{ng} / \mathrm{mL})$ restored cisplatininduced HEI-OC1 cell ototoxicity.

- A666-DEX-NP effectively preserved OHCs and showed significant hearing protection at 4,8 , and $16 \mathrm{kHz}$ by histological and functional analysis.

\section{Conclusion}

In summary, we successfully developed OHC-targeted nanoparticulate DDS driven by an A666 peptide with a sustained release profile and also assessed $\mathrm{OHCs}$ accumulation in vitro and in vivo. A666-DEX-NP demonstrated statically enhanced cytotoxicity and a protective effect against CDDP exposure via inhibition of ROS regeneration. This phenomenon could be attributed to the A666-prestin-mediated OHCs target recognition and internalization. The sustained release and accumulated concentration of DEX in cells also inhibited the apoptotic features of HEI-OC1, including nuclear condensation and the cleavage of caspase-3 against CDDP exposure. Taken together, the A666 peptide decoration and long circulating time endowed A666-DEX-NP with precise, efficient, and rapid in vivo OHCs targeting. Eventually, A666-DEX-NP showed the significantly improved protection efficiency of DEX against CDDP-induced hearing loss in guinea pigs at 4,8 , and $16 \mathrm{kHz}$ in contrast to DEX and DEX-NP.

\section{Acknowledgments}

Xueling Wang and Yuming Chen are co-first authors for this study. This work was supported by the National Natural Science Foundation of China (number 81700899), Shanghai Municipal Education Commission - Gaofeng Clinical Medicine Grant Support (number 20171919), Shanghai Health and Family Planning Commission (number 201740005), the State Key Program of National Natural Science Foundation of China (number 81330023), and the Shanghai Key Laboratory of Translational Medicine on Ear and Nose Diseases (number 14DZ2260300).

\section{Disclosure}

The authors report no conflicts of interest in this work.

\section{References}

1. Rademaker-Lakhai JM, Crul M, Zuur L, et al. Relationship between cisplatin administration and the development of ototoxicity. J Clin Oncol. 2006;24(6):918-924.

2. Manohar S, Leung N. Cisplatin nephrotoxicity: a review of the literature. J Nephrol. 2018;31(1):15-25.

3. Gregg RW, Molepo JM, Monpetit VJ, et al. Cisplatin neurotoxicity: the relationship between dosage, time, and platinum concentration in neurologic tissues, and morphologic evidence of toxicity. J Clin Oncol. 1992; 10(5):795-803. 
4. Marshak T, Steiner M, Kaminer M, Levy L, Shupak A. Prevention of cisplatin-induced hearing loss by intratympanic dexamethasone: a randomized controlled study. Otolaryngol Head Neck Surg. 2014;150(6): 983-990.

5. Al-Noury K. Distortion product otoacoustic emission for the screening of cochlear damage in children treated with cisplatin. Laryngoscope. 2011; 121(5):1081-1084.

6. Xie J, Talaska AE, Schacht J. New developments in aminoglycoside therapy and ototoxicity. Hear Res. 2011;281(1-2):28-37.

7. Guthrie OW. Aminoglycoside induced ototoxicity. Toxicology. 2008; 249(2-3):91-96.

8. Hazlitt RA, Min J, Zuo J. Progress in the development of preventative drugs for cisplatin-induced hearing loss. J Med Chem. 2018; 61(13):5512-5524

9. Leitner MG, Halaszovich CR, Oliver D. Aminoglycosides inhibit KCNQ4 channels in cochlear outer hair cells via depletion of phosphatidylinositol(4,5)bisphosphate. Mol Pharmacol. 2011;79(1): 51-60.

10. Callejo A, Sedó-Cabezón L, Juan ID, Llorens J. Cisplatin-induced ototoxicity: effects, mechanisms and protection strategies. Toxics. 2015; 3(3):268-293.

11. Liberman MC, Gao J, He DZ, Wu X, Jia S, Zuo J. Prestin is required for electromotility of the outer hair cell and for the cochlear amplifier. Nature. 2002;419(6904):300-304.

12. Dallos P, Wu X, Cheatham MA, et al. Prestin-based outer hair cell motility is necessary for mammalian cochlear amplification. Neuron. 2008;58(3):333-339.

13. Dallos P, Fakler B, Prestin FB. Prestin, a new type of motor protein. Nat Rev Mol Cell Biol. 2002;3(2):104-111.

14. Surovtseva EV, Johnston AH, Zhang W, et al. Prestin binding peptides as ligands for targeted polymersome mediated drug delivery to outer hair cells in the inner ear. Int J Pharm. 2012;424(1-2):121-127.

15. Haake SM, Dinh CT, Chen S, Eshraghi AA, van de Water TR. Dexamethasone protects auditory hair cells against TNF $\alpha$-initiated apoptosis via activation of $\mathrm{PI} 3 \mathrm{~K} / \mathrm{Akt}$ and NFאB signaling. Hear Res. 2009;255(1-2):22-32.

16. Palmer RM, Bridge L, Foxwell NA, Moncada S. The role of nitric oxide in endothelial cell damage and its inhibition by glucocorticoids. $\mathrm{Br} \mathrm{J}$ Pharmacol. 1992;105(1):11-12.

17. Özel HE, Özdoğan F, Gürgen SG, Esen E, Genç S, Selçuk A. Comparison of the protective effects of intratympanic dexamethasone and methylprednisolone against cisplatin-induced ototoxicity. J Laryngol Otol. 2016;130(3):225-234.

18. Youm I, West MB, Li W, du X, Ewert DL, Kopke RD. siRNA-loaded biodegradable nanocarriers for therapeutic MAPK1 silencing against cisplatin-induced ototoxicity. Int J Pharm. 2017;528(1-2):611-623.

19. Yoon JY, Yang KJ, Kim DE, et al. Intratympanic delivery of oligoarginine-conjugated nanoparticles as a gene (or drug) carrier to the inner ear. Biomaterials. 2015;73:243-253.
20. Agrahari V, Agrahari V, Mitra AK. Nanocarrier fabrication and macromolecule drug delivery: challenges and opportunities. Ther Deliv. 2016;7(4):257-278.

21. Kalinec G, Thein P, Park C, Kalinec F. HEI-OC1 cells as a model for investigating drug cytotoxicity. Hear Res. 2016;335:105-117.

22. Olivier JC, Huertas R, Lee HJ, Calon F, Pardridge WM. Synthesis of pegylated immunonanoparticles. Pharm Res. 2002;19(8):1137-1143.

23. Sun $C$, Wang $X$, Zheng Z, et al. A single dose of dexamethasone encapsulated in polyethylene glycol-coated polylactic acid nanoparticles attenuates cisplatin-induced hearing loss following round window membrane administration. Int J Nanomedicine. 2015;10: 3567-3379.

24. Fernandez R, Harrop-Jones A, Wang X, Dellamary L, Lebel C, Piu F. The sustained-exposure dexamethasone formulation OTO-104 offers effective protection against cisplatin-induced hearing loss. Audiol Neurootol. 2016;21(1):22-29.

25. Martín-Saldaña S, Palao-Suay R, Aguilar MR, Ramírez-Camacho R, San Román J. Polymeric nanoparticles loaded with dexamethasone or $\alpha$-tocopheryl succinate to prevent cisplatin-induced ototoxicity. Acta Biomater. 2017;53:199-210.

26. Lin LM, Colby GP, Bender MT, et al. Use of the 0.027-inch VIA microcatheter for delivery of Pipeline Flex: a technical note. J Neurointerv Surg. 2017;9(7):689-693.

27. Wen X, Ding S, Cai H, et al. Nanomedicine strategy for optimizing delivery to outer hair cells by surface-modified poly(lactic/glycolic acid) nanoparticles with hydrophilic molecules. Int J Nanomedicine. 2016;11:5959-5969.

28. Kim DK, Park SN, Park KH, et al. Development of a drug delivery system for the inner ear using poly(amino acid)-based nanoparticles. Drug Deliv. 2015;22(3):367-374.

29. Oswald M, Geissler S, Goepferich A. Determination of the activity of maleimide-functionalized phospholipids during preparation of liposomes. Int J Pharm. 2016;514(1):93-102.

30. Feng L, Wu H, Ma P, Mumper RJ, Benhabbour SR. Development and optimization of oil-filled lipid nanoparticles containing docetaxel conjugates designed to control the drug release rate in vitro and in vivo. Int J Nanomedicine. 2011;6:2545-2556.

31. Rybak LP, Mukherjea D, Jajoo S, Ramkumar V. Cisplatin ototoxicity and protection: clinical and experimental studies. Tohoku J Exp Med. 2009;219(3):177-186

32. Chirtes F, Albu S. Prevention and restoration of hearing loss associated with the use of cisplatin. Biomed Res Int. 2014;2014:925485.

33. Abi-Hachem RN, Zine A, van de Water TR. The injured cochlea as a target for inflammatory processes, initiation of cell death pathways and application of related otoprotectives strategies. Recent Pat CNS Drug Discov. 2010;5(2):147-163.
International Journal of Nanomedicine

\section{Publish your work in this journal}

The International Journal of Nanomedicine is an international, peerreviewed journal focusing on the application of nanotechnology in diagnostics, therapeutics, and drug delivery systems throughout the biomedical field. This journal is indexed on PubMed Central, MedLine, CAS, SciSearch $®$, Current Contents $\AA /$ Clinical Medicine,

\section{Dovepress}

Journal Citation Reports/Science Edition, EMBase, Scopus and the Elsevier Bibliographic databases. The manuscript management system is completely online and includes a very quick and fair peer-review system, which is all easy to use. Visit http://www.dovepress.com/ testimonials.php to read real quotes from published authors. 